\title{
ÉVOLUTION DES CARACTÉRISTIQUES PHYSICO-CHIMIQUES DE DEUX ZONES DE FRAYĖRES À SÉDIMENTATION CONTRASTÉE ET PREMIERS RÉSULTATS DE SURVIE EMBRYO-LARVAIRE DE TRUITE COMMUNE (SALMO TRUTTA).
}

\author{
F. MASSA (1), C. GRIMALDI (1), J.L. BAGLINIÈRE (2) et P. PRUNET (3)
}

(1) INRA, Laboratoire de Science du Sol, 65 rue de St-Brieuc, 35042 Rennes Cedex, France.

(2) INRA, Laboratoire d'Ecologie Aquatique, 65 rue de St-Brieuc, 35042 Rennes Cedex, France.

(3) INRA, Laboratoire de Physiologie des Poissons, Campus de Beaulieu, 35042 Rennes Cedex, France.

\section{RÉSUMÉ}

L'impact chimique et physique de la sédimentation sur la survie d'oeufs de Salmonidés a été étudié dans la partie amont de deux ruisseaux d'un même bassin à production salmonicole contrastée : le Moulinet qui présente une faible population de truites et la Roche qui est un ruisseau très productif. Les matières en suspension et les sédiments déposés ont été collectés à un pas de temps de 8 ou 15 jours en 1996 et 1997 et les survies embryo-larvaires ont été estimées en parallèle, dans des incubateurs artificiels. Les taux de survie ont été déterminés à l'émergence en 1996 et de la fécondation à l'éclosion, puis de l'éclosion à l'émergence en 1997. Les paramètres chimiques de l'eau interstitielle (oxygène, $\mathrm{pH}$, nitrates, nitrites et ammoniac) ont été mesurés au pas de temps de la semaine pendant la période d'incubation 1997 sur des dispositifs enfouis dans le substrat du ruisseau et au moment de l'éclosion dans les incubateurs contenant les oeufs.

Les premiers résultats montrent que les taux de survie embryo-larvaires sont nuls en 1996 et faibles en 1997 ( 0 à $11 \%$, moyenne : 3,3\%) dans le ruisseau du Moulinet alors que parallèlement, la survie est de $20 \%$ sur un incubateur en 1996 et varie de 0 à $23 \%$ en 1997 (moyenne : 7,7\%) dans le ruisseau de la Roche. De l'éclosion à l'émergence, les survies sont supérieures à $80 \%$ dans les deux ruisseaux.

La sédimentation est contrastée sur les deux sites, plus forte en masse dans le ruisseau de la Roche pendant la période d'incubation mais de granulométrie beaucoup plus grossière que dans le ruisseau du Moulinet (sableuse dominante contre argilolimoneuse). Les teneurs en oxygène dissous mesurées dans le substrat de la Roche restent élevées pendant toute la période d'incubation (teneur minimum de $5 \mathrm{mg} / \mathrm{l}$ ) et sont significativement supérieures à celles mesurées dans le ruisseau du Moulinet pendant la deuxième partie de l'incubation 1997. D'autre part, les teneurs en nitrates sont équivalentes à celles de l'eau de surface pour la Roche alors qu'elles sont significativement différentes de l'eau de surface pour le Moulinet. Les concentrations en nitrites et ammoniac recensées sont au maximum de $12,9 \mu \mathrm{g} / \mathrm{l}$ de $\mathrm{N}-\mathrm{NO}_{2}$ et $2 \mu \mathrm{g} / \mathrm{l}$ de $\mathrm{N}-\mathrm{NH}_{4}$ 
sur le Moulinet et de $11,4 \mu \mathrm{g} / \mathrm{l}$ de $\mathrm{N}-\mathrm{NO}_{2}$ et $1,4 \mu \mathrm{g} / \mathrm{l}$ de $\mathrm{N}-\mathrm{NH}_{4}$ pour la Roche. Les mortalités plus élevées sur le site du Moulinet pourraient être dues à une synergie de facteurs défavorables présents dans le milieu tels que la présence de faibles teneurs en oxygène et de nitrites, comme cela a été reporté dans la littérature pour des poissons adultes.

Mots-clés : sédimentation, survie embryo-larvaire, salmonidé, oxygène, nitrite, ammoniac.

\title{
PHYSICAL AND CHEMICAL TEMPORAL VARIATIONS IN TWO SPAWNING AREAS WITH CONTRASTED SEDIMENTATION DYNAMICS AND PRELIMINARY RESULTS ON THE EARLY LIFE SURVIVAL OF BROWN TROUT (SALMO TRUTTA).
}

\begin{abstract}
The effects of fines and water quality on incubating salmonid embryos were studied in two tributaries of the river Oir (Normandy, France) with different fish populations. Brown trout are very abundant in la Roche stream and their density is low in le Moulinet stream. We collected suspended and deposited sediments circulating within and sealing the substrate of two reproductive areas every 8 to 15 days in 1996 and 1997. Incubators were buried in the streambed to estimate the survival from egg to fry in 1996, and from fertilization to hatching and from hatching to emergence in 1997. The characteristics of the interstitial water (oxygen, $\mathrm{pH}$, nitrates, nitrites and ammonia) were measured once a week in 1997. This was performed with sampling tubes buried in the substratum and with a sample collected in each incubator at hatching time.
\end{abstract}

Preliminary results indicated that the amount of sediment in the spawning area was higher in la Roche stream during the incubation period. The particle size distribution was mainly sandy while it was silty in le Moulinet stream. No survivors were collected in fry traps at emergence in 1996 and between 0 and $11 \%$ (mean : $3.3 \%$ ) were found in 1997 in le Moulinet stream. The survival in one fry trap was $20 \%$ in 1996 in la Roche stream, between 0 and $23 \%$ in 1997 (mean : $7.7 \%$ ). The hatching-to-emergence survival was high in the two rivers ( 83.6 and $82.3 \%$ ), with no rain falling during this period. Dissolved oxygen concentration of the interstitial water was significantly lower in le Moulinet stream $(P=0.01)$ during the second part of the incubation period while it remained quite high in la Roche stream (minimum value of $5 \mathrm{mg} / \mathrm{l}$ ). Nitrate concentration is significantly different from that in the surface water $(P=0.01)$ in le Moulinet stream and equivalent in la Roche stream. Concentrations of nitrites and ammonia were low in the interstitial water of the two streams (maximum values : $12.9 \mu \mathrm{g} / \mathrm{N} \mathrm{N}-\mathrm{NO}_{2}$ and $240 \mu \mathrm{g} / \mathrm{l} \mathrm{N}-\mathrm{NH}_{3}$ in le Moulinet stream, $11.4 \mu \mathrm{g} / \mathrm{l} \mathrm{N}-\mathrm{NO}_{2}$ and $1.4 \mu \mathrm{g} / \mathrm{l} \mathrm{N}-\mathrm{NH}_{3}$ in la Roche stream). The higher mortalities observed in le Moulinet stream could be caused by a synergism of unfavourable factors such as low concentrations of oxygen and nitrites.

Key-words : sedimentation, early life survival, brown trout, oxygen, nitrite, ammonia.

\section{INTRODUCTION}

Le suivi des populations de Salmonidés réalisé depuis 1983 dans un bassin versant de première catégorie de Basse-Normandie a clairement mis en évidence, malgré les variations interannuelles, un faible niveau de production pour le saumon atlantique (Salmo 
salar) et une diminution des effectifs de truite commune (Salmo trutta) (BAGLINIĖRE et al., 1997). L'analyse détaillée des périodes critiques de développement a montré pour le saumon un faible taux de survie de l'oeuf au smolt comparé aux moyennes européennes ou canadiennes (PRÉVOST et al., 1996) alors que les taux de survie hivernaux du stade alevin automnal $\left(\mathrm{O}_{+}\right)$au stade juvénile de printemps $(1+)$ sont égaux, voire supérieurs (BAGLINIĖRE, données non publièes). Un faible taux de survie durant la première année de vie a également été observé chez la truite lors d'expériences de repeuplement sur un ruisseau dégradé du bassin. La survie d'alevins pré-estivaux est bonne entre juin et octobre alors que les effectifs en truitelles chutent les années suivantes à l'arrêt du déversement. Ces observations mettent en évidence l'importance des premières semaines de vie de l'embryon. Elles conditionnent le nombre d'alevins émergents et déterminent la participation des affluents au recrutement et, finalement, le renouvellement des générations à l'échelle de tout le bassin (MAISSE et BAGLINIĖRE, 1991).

Le dépôt excessif de sédiments sur les sites de frayères est l'une des causes importantes de mortalité des embryons de Salmonidés enfouis sous le gravier, car il diminue la circulation interstitielle et le renouvellement de l'oxygène dissous dans l'environnement des embryons (MEEHAM et SWANSTON, 1977 ; MARTY et al., 1986 ; EVEREST et al., 1987 ; CHAPMAN, 1988) et peut créer une barrière physique à l'émergence (CHAPMAN, 1988). L'origine du colmatage est liée à la fois aux caractéristiques du site et aux activités humaines sur les versants, notamment aux pratiques agricoles qui ont considérablement augmenté la circulation superficielle de l'eau et l'érosion des sols. La conséquence directe est un enrichissement des eaux superficielles en nitrates et une quantité plus importante de particules minérales et organiques déversées dans les rivières (GIOVANNI, 1996). La création d'un milieu anaérobie par le colmatage pourrait donc ajouter une toxicité chimique préjudiciable au développement des oeufs lors de la transformation des formes azotées présentes dans le milieu, minéralisation de la matière organique ou dénitrification des nitrates présents. Or, il n'existe pas d'étude en milieu naturel prenant en compte à la fois les mesures de polluants dans l'eau et l'analyse de la survie embryo-larvaire de Salmonidés.

Dans le Massif Armoricain où l'agriculture intensive domine, les écosystèmes aquatiques prépondérants sont ceux à Salmonidés. Ces cours d'eau, particulièrement vulnérables à l'apport de particules fines, sont donc des lieux d'étude privilégiés pour comprendre l'impact des sédiments et de la qualité de l'eau sur les conditions de développement des oeufs. Les sites choisis sont deux ruisseaux de production piscicole contrastée où l'évolution de la physico-chimie de deux zones de frayères a été suivie en parallèle avec des expérimentations de survie sous graviers. La truite a été choisie comme espèce cible car elle est présente sur l'ensemble du bassin et notamment des petits affluents où sa reproduction a lieu.

L'objectif poursuivi est d'analyser l'origine des mortalités observées au stade embryo-larvaire et de répondre aux questions suivantes : quelle est la quantité de matières fines transportées et déposées à l'échelle de la zone de frayères et quel rôle joue la géomorphologie du cours d'eau sur la nature et le dépôt de ces particules ? L'effet des sédiments sur la survie embryo-larvaire est-il uniquement physique ou un effet chimique se surajoute-t-il ? Peut-on identifier un stade critique de développement où l'embryon est davantage sensible aux conditions de l'environnement? L'étude réalisée sur ces deux bassins ne prétend pas répondre définitivement à ces questions mais apporter des éléments d'information sur la dynamique de colmatage et ses conséquences chimiques en milieu agricole. II sera ensuite nécessaire de confirmer que les facteurs de mortalité suggérés par ce travail ont des effets sur la survie embryo-larvaire de la truite. 


\section{MATÉRIEL ET MÉTHODES}

\section{Présentation du site}

Les observations ont été effectuées en 1996 et 1997 sur le Moulinet et la Roche, deux ruisseaux appartenant au réseau hydrographique de l'Oir situé en Basse-Normandie (Figure 1). L'Oir est un affluent de la Sélune qui se jette dans la baie du Mont-Saint-Michel $8 \mathrm{~km}$ après la confluence. II est long de $19,5 \mathrm{~km}$, mesure de 2 à 5 mètres de large et draine un bassin de $87 \mathrm{~km}^{2}$ essentiellement schisteux avec quelques enclaves granitiques entourées de roches cornéennes issues du métamorphisme de contact (LANGEVIN et al., 1984).

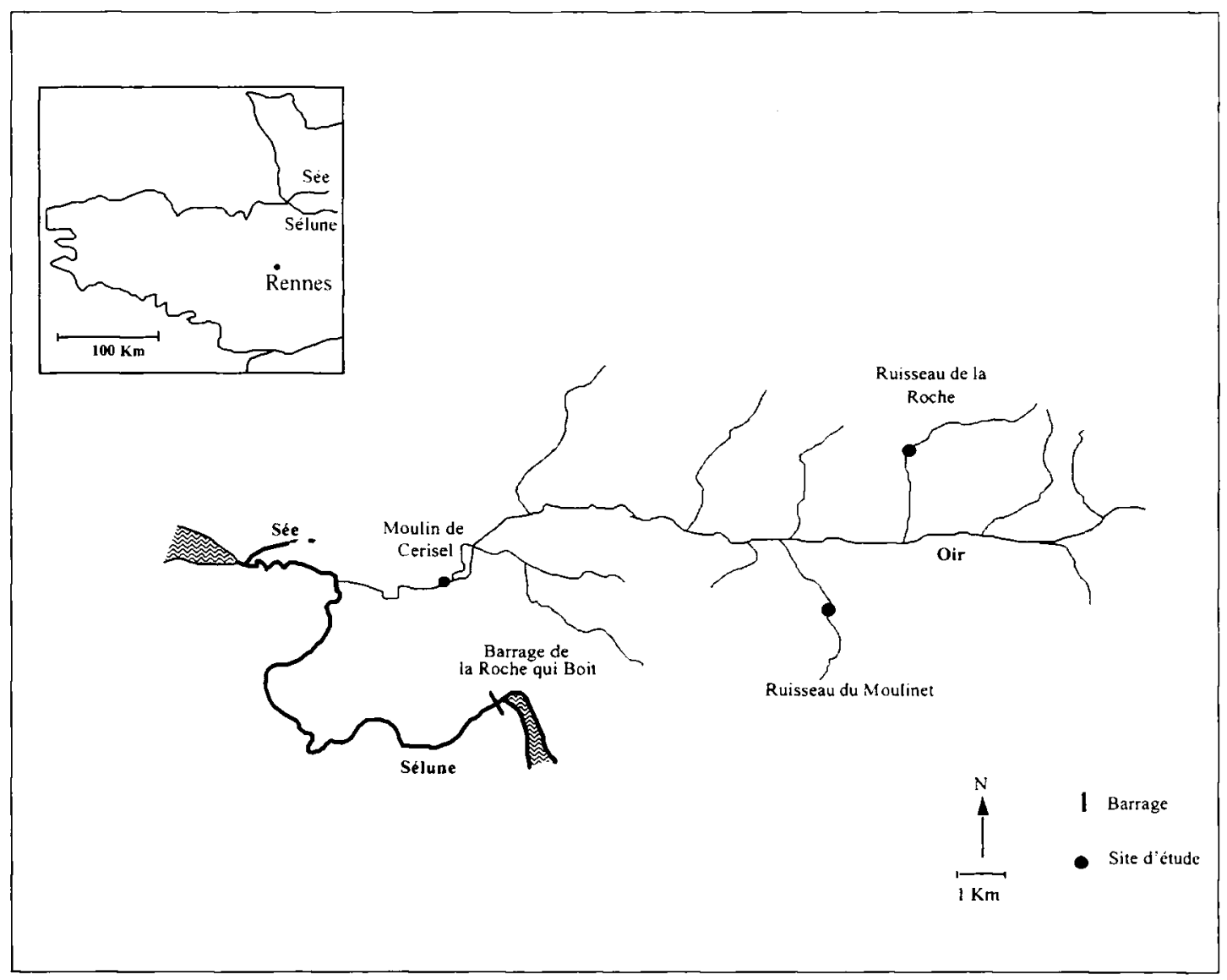

Figure 1

Localisation géographique de l'Oir et des sites étudiés.

Figure 1

Geographical situation of the river Oir and of study reaches.

Les caractéristiques générales des deux cours d'eau étudiés sont regroupées dans le Tableau I. L'intégralité du ruisseau du Moulinet coule sur schiste alors que le ruisseau de la Roche prend sa source sur granite, puis coule sur cornéenne et enfin sur schiste. Le tronçon, où les expériences de survie ont été réalisées, est situé sur cornéenne. 
Tableau 1

Caractéristiques géomorphologiques des deux cours d'eau et principales caractéristiques chimiques des deux sites étudiés (moyennes sur deux ans, écarts types entre parenthèses).

Table I

Physical characteristics of the rivers streams and major chemical characteristics of the study reaches (means and standard deviations (in parentheses) over two years of sampling).

\begin{tabular}{|l|c|c|}
\cline { 2 - 3 } \multicolumn{1}{c|}{} & Le Moulinet & La Roche \\
\hline Altitude à la source $(\mathrm{m})$ & 147 & 244 \\
\hline Altitude à la confluence $(\mathrm{m})$ & 40 & 68 \\
\hline Longueur $(\mathrm{km})$ & 3,9 & 3,4 \\
\hline Surface bassin versant $\left(\mathrm{km}^{2}\right)$ & 8,2 & 7,8 \\
\hline Débits à l'exutoire 1995-97 $(/ / \mathrm{s})$ & $55(12$ à 232$)$ & $59(8$ à 372) \\
\hline Pente du cours d'eau (\%) & & 2,7 (sur granite) \\
amont & & $5($ sur cornéenne) \\
aval & $1,2($ sur schiste) & $11,6(4,1)$ \\
\hline Température $\left({ }^{\circ} \mathrm{C}\right)$ & $10,5(2,7)$ & $6,99(0,28)$ \\
\hline $\mathrm{PH}$ & $6,90(0,19)$ & $10,9(2,0)$ \\
\hline $\mathrm{O}_{2}(\mathrm{mg} / \mathrm{l})$ & $10,2(1,6)$ & $225(10)$ \\
\hline $\mathrm{Conductivité}(\mu \mathrm{S} / \mathrm{cm})$ & $252(7)$ & $38,4(6,1)$ \\
\hline $\mathrm{NO}_{3}(\mathrm{mg} / \mathrm{l})$ & $34,2(5,5)$ & \\
\hline
\end{tabular}

Le bassin est soumis à un climat océanique. L'activité agricole est essentiellement tournée vers l'élevage bovin. Les sols sont occupés dans des proportions équivalentes par des prairies, des cultures de maïs et de céréales d'hiver. Les deux ruisseaux ont des teneurs élevées en nitrates pouvant atteindre $50 \mathrm{mg} / \mathrm{l}$ de $\mathrm{NO}_{3}$ pendant l'hiver.

\section{Caractéristiques physiques des secteurs d'étude}

Les tronçons étudiés ont une longueur d'environ 150 mètres et sont situés dans la partie amont des ruisseaux (Figure 1). Ils ont été choisis car ils possédaient des zones de frayères fonctionnelles, des fraies ayant été observées les années précédentes et au moment de la mise en place des incubateurs.

Les habitats des deux secteurs ont été décrits à l'étiage en septembre 1997 en adaptant et simplifiant les méthodes proposées par OMBREDANE et al. (1995) et KNAPP et MATTHEWS (1995). Les faciès correspondant à une zone d'écoulement homogène ont été délimités visuellement et définis suivant la typologie de CHAMPIGNEULLE (1978) et 
OMBREDANE et al. (1995) qui séparent les faciès en profonds, radiers, rapides, plats lents et courants sur des critères de profondeur, vitesses de courant et granulométrie. Chaque habitat a été caractérisé par sa longueur (mesure dans le ruisseau à l'aide d'un topofil), une largeur et un transect caractéristiques et la hauteur des berges. Sur les transects, la vitesse du courant et la hauteur d'eau sont mesurées tous les $10 \mathrm{~cm}$ et les classes granulométriques dominantes estimées visuellement ainsi que la présence de limon. Les caractéristiques d'habitat des deux cours d'eau étudiés ont été calculées en moyennant les différentes valeurs obtenues aux points de contact sur chaque transect. Les abreuvoirs sont répertoriés et localisés sur les deux secteurs.

Les secteurs étudiés présentent des caractéristiques morphologiques assez comparables (Tableau II). Les versants sont peu pentus et occupés par des prairies, les abreuvoirs sont présents sur les deux secteurs mais les incubateurs ont été enfouis en amont de ces abreuvoirs. Le ruisseau du Moulinet a été en partie recalibré et les berges sont encaissées sur le secteur étudié (Tableau II). La granulométrie du fond du ruisseau diverge selon le secteur, elle est plus grossière dans le ruisseau de la Roche où les galets et les pierres dominent et la présence de sédiments fins, de nature sableuse, est seulement répertoriée dans les profonds. Sur le fond du ruisseau du Moulinet, les classes granulométriques dominantes sont les pierres et les graviers pour les zones les plus rapides et le limon dans les plats lents et les profonds. Les graviers et les pierres sont dans $68 \%$ des cas recouverts par une fine couche de limon, même dans certaines zones courantes, ce qui montre une sédimentation fine dans ce ruisseau à la fin de l'été. La proportion des faciès d'écoulement varie sur chaque ruisseau (Tableau II). Les radiers et les plats lents prédominent dans le ruisseau du Moulinet alors que rapides, radiers et plats courants représentent $70 \%$ de la surface en eau sur la Roche. Les vitesses de courant se situent dans une gamme basse pour le ruisseau du Moulinet (Tableau II) avec des vitesses instantanées maximums de $46 \mathrm{~cm} / \mathrm{s}$ sur un radier alors qu'elles atteignent en plusieurs points des valeurs de $60 \mathrm{~cm} / \mathrm{s}$ dans le ruisseau de la Roche.

\section{Tableau II}

Caractéristiques mésologiques des secteurs et des faciès (nombre et pourcentage de la surface totale, vitesse moyenne et écart type).

\section{Table II}

\section{Habitat characteristics (means) of the study areas (number and $\%$ of total surface, mean velocity and standard deviation).}

\begin{tabular}{|c|c|c|c|c|c|c|c|c|}
\hline Profondeur moyenne étiage $(\mathrm{cm})$ & \multicolumn{4}{|c|}{5,2} & \multicolumn{4}{|c|}{9,7} \\
\hline Largeur moyenne $(\mathrm{m})$ & \multicolumn{4}{|c|}{0.9} & \multicolumn{4}{|c|}{0.8} \\
\hline Hauteur berges (m) & \multicolumn{4}{|c|}{0.7} & \multicolumn{4}{|c|}{0.35} \\
\hline $\begin{array}{l}\text { o de sédiments sur la classe } \\
\text { granulométrique dominante }\end{array}$ & \multicolumn{4}{|c|}{$68^{\circ} \circ$} & \multicolumn{4}{|c|}{$<1 \%$} \\
\hline Caracteristiques des facies & $\begin{array}{c}\text { Nombre } \\
(\%)\end{array}$ & $\begin{array}{c}V_{m} \text { en } \mathrm{cm} / \mathrm{s} \\
(\sigma)\end{array}$ & $V \min$ & $v \max$ & $\begin{array}{c}\text { Nombre } \\
(\%)\end{array}$ & $\begin{array}{c}V_{m} \text { en } \mathrm{cm} / \mathrm{s} \\
(\sigma)\end{array}$ & $\checkmark \min$ & $v \max$ \\
\hline Rapide & $0(0)$ & & & & $4(15)$ & 23 (4) & -3 & 77 \\
\hline Profond & $2(6)$ & $1(3)$ & -3 & 5 & $2(10)$ & $4(1)$ & 1 & 19 \\
\hline Plat lent & $5(32)$ & $7(2)$ & -1 & 15 & $5(16)$ & $11(3)$ & -3 & 33 \\
\hline
\end{tabular}




\section{Collecte et traitement des données}

Matières transportées et sédiments

Les dispositifs de collecte ont été mis en place dans le secteur où les incubateurs ont été placés, sur des zones courantes présentant des vitesses équivalentes (autour de $35 \mathrm{~cm} / \mathrm{s}$ au moment de la mise en place) et correspondant à des zones potentielles de reproduction.

\section{A- Collccteur de sédiments}

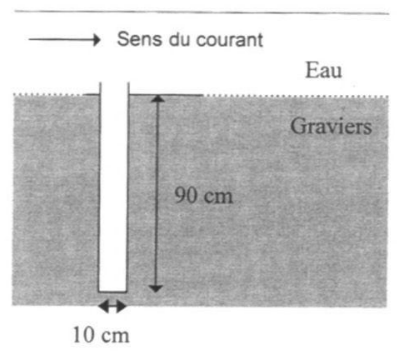

B - Collecteur de matières transportées

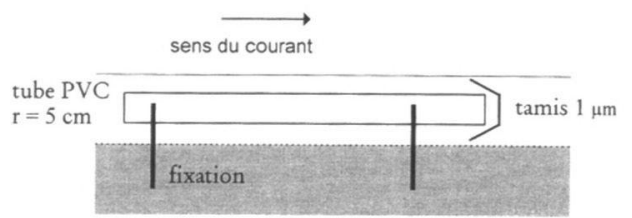

C-Collecteurs d'eau interstitielle

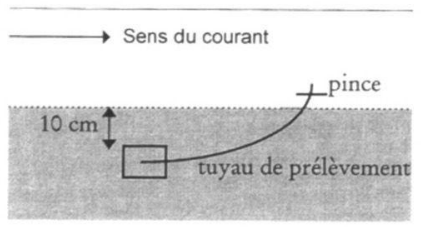

D - Incubateur

\section{Figure 2}

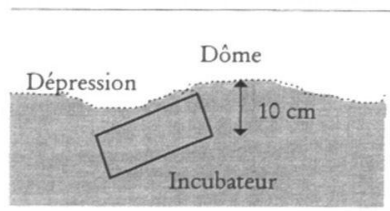

Représentation schématique des dispositifs utilisés :

A - Collecteur de sédiments (5 par site) ;

B - Collecteur de matières transportées (3 par site) ;

C - Collecteur d'eau interstitielle pour un prélèvement à l'abri de l'air (5 par site) ;

D - Incubateur.

\section{Figure 2}

Diagrams of the sampling devices :

A - Sediment trap (5 per reach) ;

B - Suspended particles trap (3 per reach) ;

C - Intertitial water sampler (5 per reach) ;

D - Incubator. 
Le premier collecteur, adapté de GIRAUD (1992), est constitué d'un cylindre de PVC de $90 \mathrm{~cm}$ de longueur et $10 \mathrm{~cm}$ de diamètre et d'une plaque de PVC disposée en collerette à l'extrémité supérieure du cylindre (Figure 2A). Le dispositif est enfoncé à la verticale dans le fond du ruisseau, la plaque de PVC reposant à l'interface eau-substrat. Son extrémité supérieure est ouverte et dépasse le fond du ruisseau de $5 \mathrm{~cm}$ pour ne pas modifier les conditions d'écoulement au niveau du collecteur (HAKANSON, 1976). Ce dispositif est constitué de telle sorte que la remise en suspension soit impossible (LAU, 1979 ; BLOESCH et BURNS, 1980), il ne permet pas d'estimer la sédimentation réelle sur les ruisseaux mais il est un indicateur du dépôt cumulé de sédiments fins apportés par le ruisseau dans la zone de frai. Les matériaux récoltés dans ces dispositifs seront identifiés sous le terme de "matières déposées ". Cinq collecteurs par site ont été installés de février à fin juillet 1997.

Le deuxième type de collecteur permet de récupérer les matières en suspension circulant dans le ruisseau. II est placé dans le sens du courant, à l'horizontale, dans l'eau de surface. II est constitué d'un tube de PVC de $130 \mathrm{~cm}$ de longueur et $10 \mathrm{~cm}$ de diamètre. A son extrémité aval est fixé un tissu-tamis de $1 \mu \mathrm{m}$ qui permet de récolter les particules da taille supérieure transitant dans le ruisseau (Figure 2B). Les particules récoltées seront appelées « matières transportées ». Trois collecteurs par site ont été mis en place de mai à fin juillet 1996 et de février à fin juillet 1997.

Les relevés sont réalisés à un pas de temps régulier pendant la période d'incubation (tous les 8 jours de février à avril) puis plus espacé pendant l'été (un prélèvement par mois). Les prélèvements sont séchés à l'étuve à $40{ }^{\circ} \mathrm{C}$ et pesés. Une analyse granulométrique à cinq fractions (argile, limons fins, limons grossiers, sables fins et sables grossiers) a été réalisée sur les particules déposées dans les collecteurs en février 1997 (période de crue) afin de comparer la nature des sédiments sur le fond des deux ruisseaux. A la même date, la teneur des sédiments en carbone organique a été mesurée par la méthode Anne et en azote par la méthode Kjeldahl.

\section{Chimie de l'eau interstitielle}

Cinq collecteurs ont été placés dans le substrat de chaque ruisseau à proximité des incubateurs afin de suivre la composition de l'eau interstitielle. Le substrat a été remanié lors de leur introduction pour simuler l'action de la truite qui lave le site de ponte en creusant la cavité où elle va déposer ses oeufs (EVEREST et al., 1987). Les collecteurs sont placés sur chaque site à une profondeur de $10 \mathrm{~cm}$ environ, ce qui correspond à la profondeur moyenne d'enfouissement des oeufs chez la truite commune (OTTAWAY et al., 1981). Ils sont constitués d'un flacon de plastique, percé de trous et recouvert d'un tissu synthétique à maille fine, sur lequel s'insère un tuyau de prélèvement fermé par une pince (Figure 2C).

Pendant la période d'incubation (15 janvier-15 avril), l'eau a été prélevée chaque semaine à l'abri de l'air à l'aide d'une seringue. $\mathrm{Le} \mathrm{pH}$, la conductivité, la température et l'oxygène dissous ont été mesurés in situ et des échantillons d'eau interstitielle ont été recueillis pour analyse au laboratoire. Ils sont filtrés à $0,45 \mu \mathrm{m}$ à l'abri de l'air, puis l'ion nitrate est dosé par chromatographie ionique et les nitrites par la réaction de Greiss. L'ammonium est dosé par spectrophotométrie de la coloration bleue d'indophénol et les concentrations en ammoniac correspondantes sont calculées en utilisant la relation d'EMERSON et al. (1975).

\section{Estimation des densités de populations de truites en place}

Les populations de truites des deux secteurs ont été inventoriées par pêche électrique en mai et octobre 1996 et 1997 par la méthode de De Lury (deux passages). Toutes les truites capturées ont été mesurées (longueur fourche en $\mathrm{mm}$ ) et des écailles 
ont été prélevées pour estimer leur âge, ce qui permet de classer les truites en juvéniles de l'année (stade $\mathrm{O}^{+}$) et truites d'un an et plus (stade $\geq 1^{+}$). Les densités ont été estimées par la méthode de SEBER et LECREN (1967) pour $100 \mathrm{~m}^{2}$ de surface en eau. La

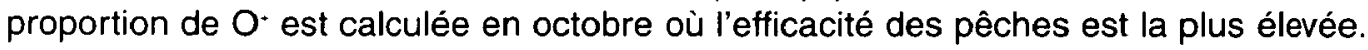

\section{Estimation des survies dans les incubateurs}

Les survies de l'oeuf à l'alevin émergent ont été estimées à l'aide d'incubateurs remplis de graviers collectés in situ et introduits à des profondeurs équivalentes dans les habitats potentiellement utilisables par la truite (zone de radier à granulométrie moyenne de 2-3 cm) (CRISP et CARLING, 1989). L'enfouissement a été réalisé par création d'un dôme et d'une dépression comme dans le cas d'une frayère naturelle (Figure 2D).

Les incubateurs utilisés ont été adaptés de BARDONNET et GAUDIN (1990). Les cagettes cylindriques grillagées de maille $1 \times 1 \mathrm{~mm}$ ont pour dimensions $30 \mathrm{~cm}$ (hauteur) $\times 14 \mathrm{~cm}$ (diamètre) en 1996 et $23 \times 10 \mathrm{~cm}$ en 1997. Les introductions ont été réalisées quelques heures après la fécondation et acclimatation des oeufs à la température de l'eau du ruisseau. Des thermographes placés dans chaque ruisseau pour un enregistrement en continu de la température ont permis de connaître le stade de développement et de déterminer la date du relevé (environ 420 degrés jours pour l'éclosion et 720 degrés jours pour l'émergence). Au moment du relevé, les incubateurs ont été plongés immédiatement dans un seau pour conserver le maximum des éléments fins qu'ils contenaient. Ils ont été ensuite vidés avec précaution, les particules solides ont été triées, puis pesées au laboratoire et le matériel biologique présent a été soigneusement décrit : nombre d'alevins éclos, vivants/morts, stade de développement, oeufs en décomposition... Le contrôle de la qualité des oeufs a été réalisé chaque année en conservant un lot témoin incubé en conditions optimales à la pisciculture du Moulin de Cerisel, située sur l'Oir (Figure 1).

En 1996, trois incubateurs par site (100 oeufs) ont été enfouis avec des oeufs fécondés à la pisciculture de Cerisel et issus de truites sauvages capturées sur l'Oir au mois de janvier. Le relevé des incubateurs a été réalisé au stade émergent au mois d'avril.

En janvier 1997, les incubateurs ont été enfouis avec des oeufs fécondés provenant de la pisciculture de la Gouarnay (Fédération de Pêche du Morbihan) où la fécondation a eu lieu. Sept incubateurs par site (100 oeufs par piège) ont été enfouis à la fécondation et relevés à l'éclosion (12 mars). Un tube a été placé dans chaque incubateur pour permettre le prélèvement d'eau interstitielle et la mesure de l'oxygène dissous, du $\mathrm{pH}$ et de la température au moment de l'éclosion. Une deuxième série de trois incubateurs a été mise en place sur chaque site au mois de mars (50 alevins éclos de pisciculture par piège) et relevée mi-avril à l'émergence.

\section{RÉSULTATS}

\section{Qualité chimique du milieu interstitiel}

Dans les collecteurs, le suivi de la teneur en oxygène dissous montre une diminution assez constante et progressive dans l'eau interstitielle sur les deux cours d'eau comparable jusqu'au 4/3/97 (Figure 3). Puis, elle devient significativement plus forte dans le ruisseau du Moulinet (test de $t, P \leq 0,01$ ) où elle atteint des valeurs faibles de 1,8 à $3 \mathrm{mg} / \mathrm{l}$. La variabilité spatiale est importante sur un même site. Les teneurs oscillent entre 3,9 et $8,3 \mathrm{mg} / \mathrm{l}$ dans le ruisseau du Moulinet et entre 5,2 et $10,8 \mathrm{mg} / \mathrm{l}$ sur celui de la Roche à la date du 24/2/97. 


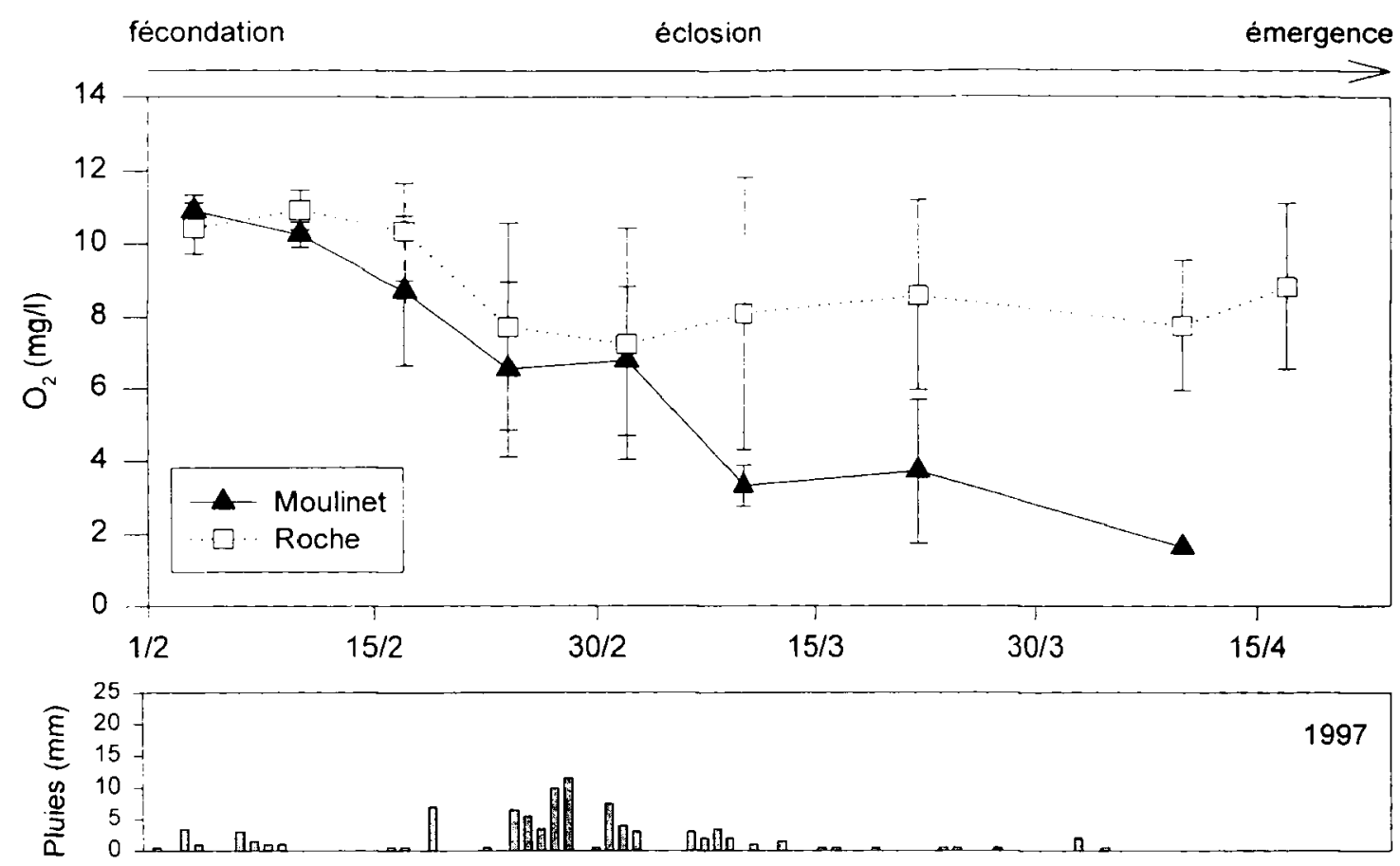

Figure 3

Variation de la teneur en oxygène dissous dans les collecteurs d'eau interstitielle pendant la période d'incubation 1997 (valeurs moyennes et écarts types sur 4 à 5 prélèvements excepté à la date du 11/4/97 (un prélèvement pour le ruisseau du Moulinet).

\section{Figure 3}

Temporal trends of dissolved oxygen concentration during the incubation period in interstitial water samples (means and standard deviations of 4 to 5 samples on the two study sites except for the 11/4/97 in le Moulinet (one sample)).

Les concentrations en nitrates évoluent peu au cours du temps, de 17 à $24 \mathrm{mg} / \mathrm{l}$ pour l'eau interstitielle sur le Moulinet et de 38 à $46 \mathrm{mg} / \mathrm{l}$ pour le ruisseau de la Roche. Elles sont assez proches de celles du ruisseau mais presque toujours inférieures sur le secteur de la Roche alors qu'il existe une différence significative entre l'eau interstitielle et l'eau du ruisseau sur le secteur du Moulinet (test de $t, P \leq 0,01$ ). Les teneurs en ammonium varient de 32 à $240 \mu \mathrm{g} / \mathrm{l}$ de $\mathrm{N}^{-} \mathrm{NH}_{4}$ dans le Moulinet et de 6 à 190 dans la Roche, ce qui correspond à des valeurs de 0,04 et $2 \mu \mathrm{g} / \mathrm{l}$ de $\mathrm{N}-\mathrm{NH}_{3}$ pour le Moulinet et à 0,05 et $1,4 \mu \mathrm{g} / \mathrm{l}$ de $\mathrm{N}-\mathrm{NH}_{3}$ pour celui de la Roche en prenant en compte les valeurs de $\mathrm{pH}$ et de température au moment du relevé. La variabilité spatiale est forte entre les collecteurs d'eau d'un même site. Les concentrations en nitrites s'échelonnent entre 4 et $12,9 \mu \mathrm{g} / \mathrm{l}$ de $\mathrm{N}-\mathrm{NO}_{2}$ pour le Moulinet et entre 3,5 et 11,4 $\mu \mathrm{g} / \mathrm{l}$ de $\mathrm{N}-\mathrm{NO}{ }_{2}$ pour la Roche. Les variations observées dans les deux ruisseaux ne présentent pas de différence significative. Le $\mathrm{pH}$ baisse progressivement pour les deux ruisseaux, de 7,13 à 6,70 pour la Roche et de 7,31 à 6,65 pour le Moulinet, rendant le milieu légèrement acide.

Dans les incubateurs, les teneurs en oxygène dissous mesurées à l'éclosion sont variables mais élevées, elles oscillent entre 6,4 et $9,7 \mathrm{mg} / \mathrm{l}$ dans le ruisseau de la Roche (moyenne : $8,0 \mathrm{mg} / \mathrm{l}$ ) et entre 3,3 et 9 sur le Moulinet (moyenne : $6,7 \mathrm{mg} / \mathrm{l}$ ). Le $\mathrm{pH}$ mesuré dans les incubateurs est de 6,65 pour le Moulinet et de 6,61 sur la Roche en moyenne, l'écart type entre les valeurs étant très faible (Tableau IV). 


\section{Tableau IV}

Composition granulométrique $(\mathrm{g} / \mathrm{kg})$ et teneurs en carbone et azote $(\mathrm{g} / \mathrm{kg})$ des fractions inférieures ou égales à $2 \mathrm{~mm}$ sur les matières transportées lors du prélèvement du 17/2/97.

Table IV

Particle size distribution and amounts of organic matter in suspended sediments ( $\leq 2 \mathrm{~mm}$ ) for the 17/2/97 sample.

\begin{tabular}{|c|c|c|c|c|}
\hline & \multicolumn{2}{|c|}{ Le Moulinet } & \multicolumn{2}{|c|}{ La Roche } \\
\hline & masse $(\mathrm{g} / \mathrm{kg})$ & $\sigma$ & masse $(g / k g)$ & $\sigma$ \\
\hline Fraction $<2 \mu \mathrm{m}$ & 119,4 & $(30,6)$ & 61,2 & $(5,6)$ \\
\hline de $2-20 \mu m$ & 174 & $(33,6)$ & 210,8 & $(54,5)$ \\
\hline de $20-50 \mu \mathrm{m}$ & 239,2 & $(54,6)$ & 67,2 & $(5,1)$ \\
\hline de $50-200 \mu^{m}$ & 138 & $(20,7)$ & 129,8 & $(13,9)$ \\
\hline de $200-2000 \mu \mathrm{m}$ & 329,4 & $(130,6)$ & 531 & (74) \\
\hline Carbone organique & 33,8 & $(9,1)$ & 14,8 & $(2,1)$ \\
\hline Matière organique & 58,2 & $(15,6)$ & 25,5 & $(3,5)$ \\
\hline Azote organique & 2,4 & $(0,6)$ & 1,2 & $(0,2)$ \\
\hline Rapport C/N & 13,8 & $(1,3)$ & 12,1 & $(0,4)$ \\
\hline
\end{tabular}

\section{Matières transportées et déposées dans la zone de frayères}

Le suivi des matières transportées dans les ruisseaux montre une dynamique saisonnière claire et contrastée sur les deux sites (Figure 4). De fin avril à juillet 1996, elles sont en quantité jusqu'à trois fois plus importantes dans le ruisseau du Moulinet. La tendance inverse est observée au cours de l'hiver 1997, les valeurs relevées étant deux fois plus importantes dans le ruisseau de la Roche, avec toutefois une exception en février qui correspond à la seule période de précipitations dépassant $10 \mathrm{~mm}$ pendant la période d'incubation.

II existe une corrélation forte entre la pluviométrie mensuelle et la quantité de matières transportées pendant la même période pour les deux années dans le ruisseau du Moulinet $(r=0,90, n=8)$. Cette corrélation est faible dans le ruisseau de la Roche $(r=0,47, n=8)$.

De la même façon, les matières déposées de février à mi-mars 1997 dans le ruisseau de la Roche sont au moins trois fois plus élevées par rapport au Moulinet. La tendance est contraire en été, les plus fortes pluies à cette période semblent augmenter cette différence.

L'analyse granulométrique des sédiments déposés montre que le grain moyen des particules dans le ruisseau du Moulinet est très inférieur à celui de la Roche (Tableau IV). Les argiles et limons sont majoritaires sur le Moulinet ( $53 \%$ de la masse) alors qu'ils ne représentent que $25 \%$ dans le ruisseau de la Roche où les sables dominent. Les teneurs en matière organique sont supérieures dans le ruisseau du Moulinet $(3,4 \%$ contre $1,5 \%$ dans le ruisseau de la Roche). Le rapport $\mathrm{C} / \mathrm{N}$ est sensiblement équivalent sur les deux cours d'eau (Tableau IV). 

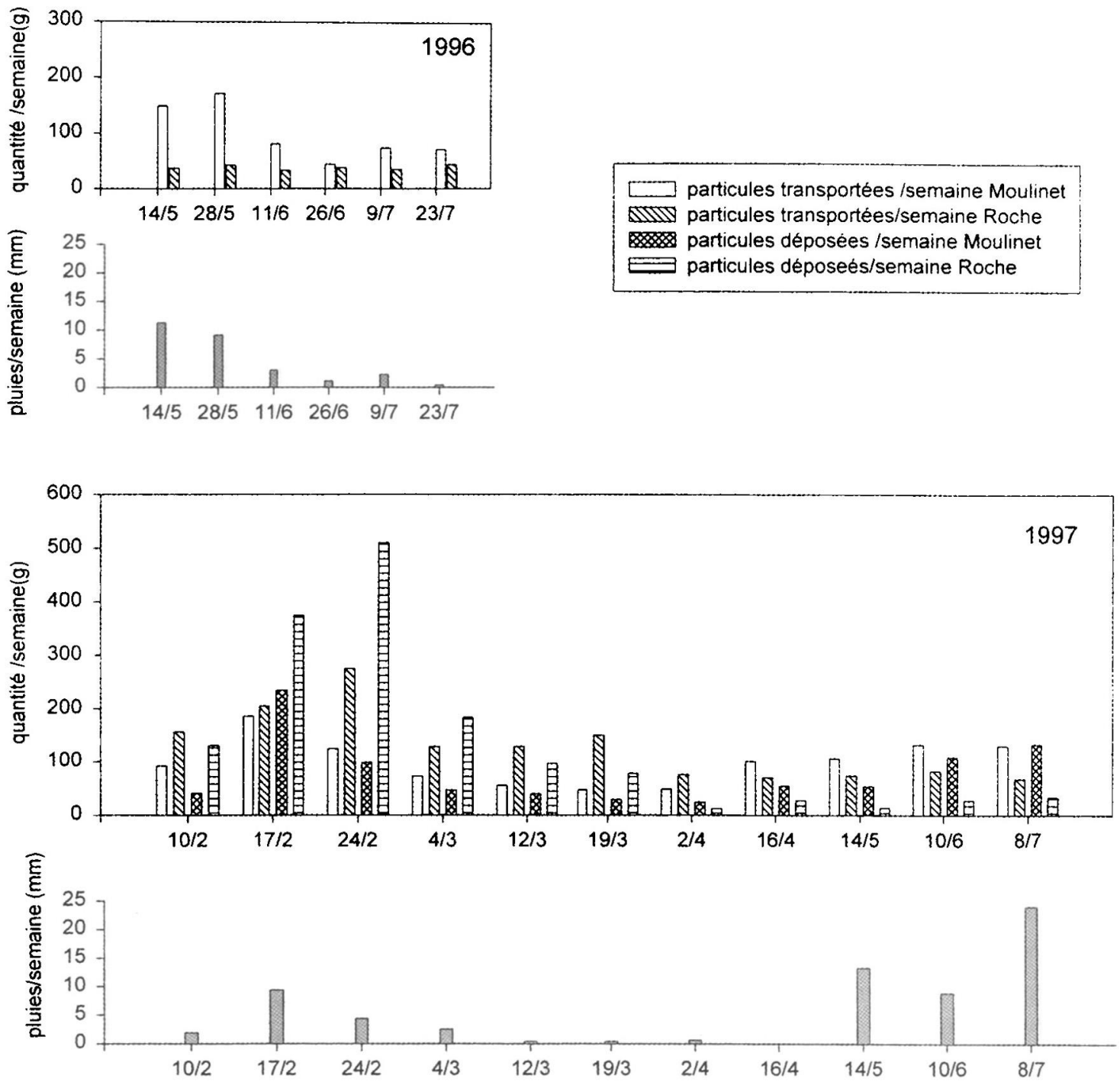

\section{Figure 4}

Suivi de la sédimentation (matières en suspension, sédiments déposés) et de la pluviométrie sur les deux secteurs (moyenne sur 3 points de prélèvements pour les matières transportées, moyennes sur 5 points de prélèvements pour les matières déposées). Les quantités mesurées à un pas de temps supérieur à la semaine sont ramenées à un intervalle de 8 jours.

\section{Figure 4}

Temporal trends of the amounts of suspended and deposited sediments on the two study reaches (means of 3 samples for suspended sediments, of 5 samples for deposited sediments). The amounts are divided by 2 or 4 when the sampling time interval is $\mathbf{2}$ weeks or 1 month. 


\section{Taux de survie sous graviers}

En 1996, le taux de survie dans le secteur du Moulinet est de $0 \%$ pour les 3 incubateurs et le développement le plus complet observé sur les embryons morts est le stade oeuf oeillé. Dans le ruisseau de la Roche, deux incubateurs ont été retrouvés ouverts au moment du relevé, l'un était vide et l'autre contenait deux alevins vivants. Le troisième incubateur, fermé, présentait un taux de survie de $20 \%$. Sur ce ruisseau, l'embryogenèse peut être complète, ce qui laisse penser que des alevins vivants ont pu s'échapper des deux autres incubateurs. Le taux de survie à l'émergence du témoin placé en pisciculture est de $84 \%$.

En 1997, les taux de survie à l'éclosion sont faibles dans les sept incubateurs. Ils varient de 0 à $11 \%$ dans le ruisseau du Moulinet et de 0 à $23 \%$ sur celui de la Roche avec une valeur moyenne de survie de $7,7 \%$ sur la Roche $(\sigma=8,1, n=7)$ et de $3,3 \%$ dans le ruisseau du Moulinet $(\sigma=4,6, n=6)$. La différence de survie entre les deux ruisseaux n'est pas significative (test de Mann-Withney, $P=0,234$ ). Le faible taux de survie relevé sur le lot témoin de pisciculture (66\% de survie à l'éclosion pour 730 oeufs incubés) montre un problème de qualité des oeufs et ne permet pas en toute rigueur la comparaison entre les sites. Le taux de survie de l'éclosion à l'émergence est en revanche très élevé sur les deux sites $(83,6 \%$ sur le Moulinet, $\sigma=5,9$ et $82,3 \%$ sur la Roche, $\sigma=7,1)$.

Les quantités de sédiments fins recueillis dans les incubateurs sont plus importantes en moyenne dans le ruisseau de la Roche $(871 \mathrm{~g}$ contre $390 \mathrm{~g}$ pour le Moulinet en 1996, $314 \mathrm{~g}(\sigma=200)$ contre $264 \mathrm{~g}(\sigma=177)$ en 1997). La variabilité est forte entre les incubateurs d'un même site.

\section{Caractéristiques des populations de truites en place}

En 1996, quelle que soit la période de l'année, les densités totales observées dans le Moulinet sont nettement inférieures à celles recensées dans le ruisseau de la Roche (Tableau V). En octobre 1996, la proportion de $\mathrm{O}^{+}$est plus faible dans le Moulinet $(45,8 \%)$ par rapport à celle observée pour la Roche $(68,4 \%)$. En 1997, la très forte augmentation de densité de $\mathrm{O}^{*}$ de truites entre le mois de mai et octobre dans le ruisseau du Moulinet met en évidence un vraisemblable déversement de juvéniles d'élevage entre les deux pêches. Ces truitelles ont un mode de taille distinct qui ne correspond pas aux conditions de croissance observées en milieu naturel et présentent une livrée caractéristique différente des individus de plus petite taille. Par ailleurs, la densité de juvéniles endogènes estimée sur le Moulinet est relativement forte en 1997, elle pourrait être liée à la faible pluviométrie hivernale.

\section{DISCUSSION}

Cette étude réalisée en milieu naturel est la première à relier l'évolution de la composition chimique de l'eau interstitielle dans la zone d'étude à la survie embryo-larvaire de la truite sur deux bassins à sédimentation contrastée.

Les premiers résultats de survie, bien que préliminaires, semblent mettre en évidence une mortalité moyenne plus forte dans le ruisseau du Moulinet. Ils sont à confirmer du fait de la mauvaise qualité de la ponte en 1997, des résultats partiels obtenus en 1996 après l'ouverture des pièges enfouis dans le ruisseau de la Roche et de la forte variabilité intra-site. Les estimations de densités, moyen de contrôle et référence du fonctionnement de la zone à l'échelle de l'année, semblent valider les résultats de survie 


\section{Tableau V}

Densités estimées (individus $/ 100 \mathrm{~m}^{2}$ ) sur les secteurs d'étude du Moulinet et de la Roche (méthode de SEBER et LECREN, 1967) au printemps et à l'automne en 1996 et 1997.

\section{Table V}

Densities of brown trout populations in the two study reaches during spring and autumn 1996 and 1997 (method by SEBER and LECREN, 1967).

\begin{tabular}{|l|r|r|r|r|}
\cline { 2 - 5 } \multicolumn{1}{c|}{} & \multicolumn{2}{c|}{ Le Moulinet } & \multicolumn{2}{c|}{ La Roche } \\
\hline Densités (ind $/ 100 \mathrm{~m}^{2}$ ) & $\mathrm{O}^{+}$ & $\geq 1^{\cdot}$ & $\mathrm{O}^{+}$ & $\geq 1^{\circ}$ \\
\hline Mai 1996 & 0,7 & 4,4 & 5,9 & 21,9 \\
\hline Octobre 1996 & 12,6 & 14,9 & 36,9 & 17 \\
\hline Mai 1997 & 10,5 & 4,9 & 36,5 & 26,8 \\
\hline Octobre 1997 & $\left(16^{*}\right.$ & 7,5 & 45,8 & 28,4 \\
\hline
\end{tabular}

" densité endogène estimée par distinction des classes de taille (la valeur entre parenthèses correspond à la densité totale tenant compte du repeuplement).

obtenus sur les secteurs étudiés. Sur la Roche, les densités sont fortes et caractéristiques d'un ruisseau productif aussi bien aux mois de mai que d'octobre et la proportion de $\mathrm{O}^{+}$très élevée sur ce cours d'eau montre son rôle de pépinière pour le recrutement à l'échelle du bassin. $A$ contrario, les densités en adultes sont faibles sur le Moulinet et la proportion de juvéniles est peu élevée pour les deux années. En 1997, le recrutement faible sur ce ruisseau est en partie masqué par des opérations de repeuplement menées au printemps. Les densités relativement élevées obtenues en octobre 1997 avec ces poissons de repeuplement confirment que ce sont les premières semaines de vie sous graviers qui sont primordiales pour le recrutement.

Ce premier suivi montre également une sédimentation contrastée sur les deux cours d'eau qui s'exprime à la fois dans la granulométrie des particules déposées et la dynamique de colmatage et de transport des matières fines. Les quantités de particules les plus importantes sont transportées et déposées en hiver, pendant la période d'incubation, et sont alors plus élevées sur la Roche. Les dispositifs mis en place ne permettent pas de connaître la remise en suspension sur le fond des ruisseaux mais ce sont vraisemblablement des particules déplacées par charriage ou saltation qui expliquent les plus fortes quantités de matières déposées dans le ruisseau de la Roche pendant les pluies, les débits plus soutenus sur granite favorisant le remaniement du fond du ruisseau. Ce phénomène est observé dans une moindre mesure dans le ruisseau du Moulinet, les particules solides plus fines étant plus facilement remises en suspension, même à de faibles débits.

La granulométrie des sédiments et du substrat sur les ruisseaux étudiés est à mettre en relation avec la nature de la roche mère, le site de la Roche reposant sur cornéenne et en amont sur granite alors que le Moulinet coule sur schiste. Son rôle sur la perméabilité intragravellaire et l'oxygénation des sites de ponte a déjà été démontré en laboratoire (VAUX, 1962 ; BESCHTA et JACKSON, 1979 ; MARTY et al., 1986 ; EVEREST et al., 1987 ) et il est confirmé dans cette étude en milieu naturel. La nature argilo-limoneuse des particules fines et la taille du matériau initial de la frayère sur le ruisseau du Moulinet favorisent l'apparition de conditions anoxiques et ceci, malgré les plus faibles quantités de 
particules transitant sur ce ruisseau. D'autre part, la disparition progressive de l'oxygène dissous rend possible la dénitrification dans ces milieux où les nitrates sont présents et la minéralisation de la matière organique en anaérobiose avec apparition de nitrites et d'ammoniac dans le milieu interstitiel (KNOWLES, 1982 ; PELMONT, 1993). Les teneurs mesurées dans cette étude sont du même ordre de grandeur que celles recensées à l'intérieur de frayères en milieu naturel par RUBIN et al. (1996) qui ne fournit pas de résultat de survie. De même, la forte variabilité observée ici a aussi été constatée en milieu naturel (PETERSON et QUINN, 1996 ; RUBIN et al., 1996).

Les doses létales en ammoniac et nitrites estimées à partir de tests réalisés en laboratoire s'échelonnent entre 0,2 et $0,4 \mathrm{mg} / \mathrm{l}$ de $\mathrm{N}_{-} \mathrm{NH}_{3}$ pour des Salmonidés de différentes tailles, le seuil de toxicité augmentant à mesure que le poisson grossit (SMITH et WILLIAMS, 1974 ; RUSSO et al., 1974, 1978 ; RICE et STOKES, 1975 ; PERRONE et MEADE, 1977). BURKHALTER et KAYA (1977) notent des retards de croissance importants, l'incidence de maladies et une hypertrophie des lamelles secondaires sur des oeufs de truite arc-en-ciel dès $0,19 \mathrm{mg} / \mathrm{l}$. Pour les nitrites, les valeurs seuils reportées sont très dépendantes de la teneur en chlorure, la LC50 $96 \mathrm{~h}$ (dose pour atteindre $50 \%$ de mortalité en 96 heures) varie de 0,25 à $6,69 \mathrm{mg} / \mathrm{l}$ de $\mathrm{N}-\mathrm{NO}_{2}$ pour des valeurs de chlorure de 0,35 à $40,9 \mathrm{mg} / \mathrm{l}$ (RUSSO et THURSTON, 1977), la sensibilité semble s'accroître au cours du développement (PERRONE et MEADE, 1977). La concentration en chlorure observée sur nos ruisseaux (environ $25 \mathrm{mg} / \mathrm{l}$ ) supposerait un seuil de toxicité en nitrites de $6 \mathrm{mg} / \mathrm{l}$ pour la truite arc-en-ciel adulte (LEWIS et MORRIS, 1986). Les concentrations en ammoniac et en nitrites mesurées in situ sont donc relativement faibles comparées aux doses létales estimées en laboratoire.

Le pas de temps retenu et le mode de prélèvement utilisé pour la mesure des paramètres chimiques de l'eau ne fournissent qu'un aperçu des conditions auxquelles sont soumis les embryons, le mélange d'eaux issues de différents microsites plus ou moins interconnectés donnant, de plus, une valeur moyenne qui nivelle la forte variabilité spatiale qui existe sur les sites. Le stade maximum de développement atteint en 1996 dans le ruisseau du Moulinet (stade oeuf oeillé observé sur les embryons morts) et les forts taux de survie estimés en 1997 entre l'éclosion et l'émergence sur ce même ruisseau semblent montrer que la période critique se situe au tout début de la période embryo-larvaire, au moment de l'apparition des yeux. Ces résultats sont en accord avec certains travaux réalisés sur les premières phases de développement des Salmonidés qui montrent une tolérance à l'hypoxie plus grande des alevins vésiculés par rapport aux oeufs (à l'exception de la période d'éclosion), leur surface respiratoire plus importante à ce stade (apparition des branchies) et leur capacité à se mouvoir leur permettant de rechercher un environnement plus riche en oxygène (ROMBOUGH, 1988). Mais, si la différence de diamètre des particules et du substrat entre les deux cours d'eau semble être la cause de la diminution de la teneur en oxygène dissous dans le milieu interstitiel, les valeurs en oxygène recensées sur le Moulinet jusqu'à l'éclosion ne sont pas suffisantes à elles seules pour expliquer les mortalités observées sur ce ruisseau.

En définitive, ces premiers résultats nous amènent à formuler l'hypothèse que les effets croisés d'une plus forte hypoxie et de la présence d'ammoniac et/ou de nitrites dans le ruisseau soumis à une sédimentation fine pourraient entraîner des mortalités fortes dès les premiers stades de développement. Les expérimentations en conditions contrôlées sur les stades de développement précoces pour tester les seuils de toxicité des formes de l'azote (nitrite et ammoniac) ont été jusqu'à présent réalisées en conditions optimales où l'oxygène n'est jamais un facteur limitant. Or, les travaux de THURSTON et al. (1981) sur la truite arc-en-ciel font état d'une forte corrélation entre le seuil de toxicité en ammoniac et la teneur en oxygène dissous dans le milieu et montrent l'importance de la synergie de ces facteurs. Le faible nombre de travaux réalisés jusqu'à présent ne permet pas de valider cette hypothèse mais des expérimentations sont actuellement en cours pour tester ces synergies. 


\section{REMERCIEMENTS}

Les auteurs remercient chaleureusement F. Rouault et F. Marchand pour leur collaboration technique et leur aide précieuse lors des déplacements sur le terrain ainsi que $V$. Forget qui a égayé ces froides journées d'hiver. Merci également à $A$. Regeard pour les analyses en chromatographie.

Ce travail a été réalisé dans le cadre d'un contrat de plan financé par le ministère de l'Environnement (programme $n^{\circ} 15$ - action 2A) sur "le rôle des facteurs du milieu dans la réponse des hydrosystèmes de Bretagne aux perturbations anthropiques " et l'action incitative de l'INRA (programme Armor).

\section{BIBLIOGRAPHIE}

BAGLINIĖRE J.L., MASSA F., MARCHAND F., 1997. Impact de l'intensification agricole sur l'état de la population de salmonidés d'un petit cours d'eau : le cas de l'Oir (Basse-Normandie). Rapport de synthèse Laboratoire d'Ecologie Aquatique, INRA Rennes, $14 \mathrm{p}$.

BARDONNET A., GAUDIN H., 1990. Diel pattern of emergence in grayling (Thymallus thymallus Linnaeus, 1758). Can. J. Zool., 68, 465-469.

BESCHTA R.L., JACKSON W.L., 1979. The intrusion of fine sediments into a stable gravel bed. J. Fish. Res. Board Can., 36, 204-210.

BLOESCH J., BURNS N.M., 1980. A critical review of sediment trap techniques. Schweiz. Z. Hydrol., 42 (1), 15-53.

BURKHALTER D.E., KAYA C.M., 1977. Effects of prolonged exposure to ammonia on fertilised eggs and sac-fry of rainbow trout (Salmo gairdneri). Trans. Am. Fish. Soc., 106 (5), 470-475.

CHAMPIGNEULLE A., 1978. Caractéristiques de l'habitat piscicole et de la population de juvéniles sauvages de saumon atlantique (Salmo salar L.) sur le cours principal du Scorff (Morbihan). Thèse $3^{\text {eme }}$ cycle Biologie Animale, Université de Rennes I, $92 \mathrm{p}$.

CHAPMAN D.W., 1988. Critical review of variables used to define effects of fines in redds of large salmonids. Trans. Am. Fish. Soc., 117, 1-21.

CRISP D.T., CARLING P.A., 1989. Observations of siting, dimensions and structure of salmonid redds. J. Fish Biol., 34, 119-124.

EMERSON K., RUSSO R.C., LUND R.E., THURSTON R.V., 1975. Aqueous equilibrium calculations : effects of $\mathrm{pH}$ and temperature. J. Fish. Res. Board Can., 32, 23792383.

EVEREST F.H., BESCHTA R.L., SCRIVENER J.C., KOSKI K.V., SEDELL J.R., CEDERHOLM C.J., 1987. Streamside Management Forestry and Fishery Interactions, Salo E.O. and Cundy T.W. (eds), Institute of Forest Resources, Washington, $141 \mathrm{p}$.

GIOVANNI R., 1996. Situation de la dégradation de la qualité des eaux courantes en Bretagne : cas des nitrates et de deux pesticides, l'atrazine et le lindane. Cybium, 20 (3) suppl., 143-162.

GIRAUD F., 1992. Modélisation hydrologique d'une zone humide agricole : perspective pour l'étude du transport d'azote et de phosphore dans le réseau hydraulique. Cas du marais de Möeze, Charente-Maritime. Thèse $3^{\text {eme }}$ cycle Evolution des systèmes naturels et modifiés, Université de Rennes I, $223 p$.

HAKANSON L., 1976. A bottom sediment trap for recent sedimentary deposits. Limnol. Oceanogr., 21 (1), 170-174. 
KNAPP R.A., MATTHEWS K.R., 1995. Livestock grazing, golden trout, and streams in the golden trout wilderness, California : impacts and management implications. N. Am. J. Fish. Manag., 16, 805-820.

KNOWLES R., 1982. Denitrification. Microbiological Reviews, 46 (1), 43-70.

LANGEVIN C., MINOUX L., L'HOMER A., LAUTRIDOU J.P., DASSIBAT C., VERRON G., 1984. Notice explicative de la feuille d'Avranches à 1/50 000, BRGM, $54 \mathrm{p}$.

LAU Y.L., 1979. Laboratory study of cylindrical sedimentation traps. J. Fish. Res. Board Can., 36, 1288-1291.

LEWIS W.M., MORRIS D.P., 1986. Toxicity of nitrite to fish : a review. Trans. Am. Fish. Soc., 115, 183-195.

MAISSE G., BAGLINIÈRE J.L., 1991. Biologie de la truite commune (Salmo trutta L.) dans les rivières françaises. In Baglinière J.L. et Maisse G., La truite : biologie et écologie, 25-45, INRA Publ., Paris.

MARTY C., BEALL E., PAROT G., 1986. Influence de quelques paramètres du milieu d'incubation sur la survie d'alevins de saumon atlantique Salmo salar L. en ruisseau expérimental. Int. Rev. Ges. Hydrobiol., 71 (3), 349-361.

MEEHAM W.R., SWANSTON D.N., 1977. Effects of gravel morphology on fine sediment accumulation and survival of incubating salmon eggs. USDA Forest Services Research Paper, 220 p.

OMBREDANE D., HAURY J., CHAPON P.M., 1995. Heterogeneity and typology of fish habitat in the main stream of a Breton coastal river (Elorn, Finistère, France). Hydrobiologia, 300/301, 259-268.

OTTAWAY E.M., CARLING P.A., CLARKE A., READER N.A., 1981. Observations of the structure of brown trout, Salmo trutta Linnaeus, redds. J. Fish. Biol., 19, 593-607.

PELMONT J., 1993. Nitrate, dénitrification. In Bactéries et environnement, adaptations physiologiques, Presses Universitaires, 576-604, Grenoble.

PERRONE S.J., MEADE T.L., 1977. Protective effect of chloride on nitrite toxicity to coho salmon (Oncorhynchus kisutch). J. Fish. Res. Board Can., 34 (4), 486-92.

PETERSON N.P., QUINN T.P., 1996. Spatial and temporal variation of dissolved oxygen in natural egg pockets of chum salmon in Kennedy Creek, Washington. J. Fish. Biol., 48, 131-143.

PRÉVOST E., BAGLINIĖRE J.L., MAISSE G., NIHOUARN A., 1996. Premiers éléments d'une relation stock/recrutement chez le saumon atlantique (Salmo salar) en France. Cybium, 20-(3), 7-26.

RICE S.D., STOKES R.M., 1975. Acute toxicity of ammonia to several developemental stages of rainbow trout, Salmo gairdneri. Fish. Bull., 73 (1), 207-211.

ROMBOUGH P.J., 1988. Respiratory gas exchange, aerobic metabolism, and effects of hypoxia during early life. In Hoar W.S. et Randall D.J., Fish physiology, 123-143, Academic Press Publ., San Diego.

RUBIN J.F., GLIMSÄTER C., 1996. Egg-to-fry survival of the sea trout in some stream of Gotland. J. Fish. Biol., 48, 585-606.

RUSSO R.C., SMITH C.E., THURSTON R.V., 1974. Acute nitrite toxicity to rainbow trout (Salmo gairdneri). J. Fish. Res. Board Can., 107 (2), 361-368.

RUSSO R.C., THURSTON R.V., 1977. The acute toxicity of nitrite to fishes. In Tubb R.A., Recent advances in fish toxicology, 118-131, U.S. Environmental Protection Agency, Ecological Research Series EPA-600/3-77-085, Corvallis, Oregon.

RUSSO R.C., THURSTON R.V., SMITH C.E., 1978. Acute toxicity of ammonia and nitrite to cutthroat trout fry. Trans. Am. Fish. Soc., 117, 1-21. 
SEBER J.A.F., LECREN E.D., 1967. Estimating population parameters from large catches relative to the population. J. Animal Ecology, 36, 631-643.

THURSTON R., PHILLIPS G.R., RUSSO R.C., 1981. Increased toxicity of ammonia to rainbow trout (Salmo gairdneri) resulting from reduced concentrations of dissolved oxygen. Can. J. Fish. Aquat. Sci., 38, 983-988.

SMITH C.E., WILLIAMS W.G., 1974. Experimental nitrite toxicity in rainbow trout and chinook salmon. Trans. Am. Fish. Soc., 2, 389-390.

VAUX W.G., 1962. Interchange of stream and intragravel water in a salmonid spawning riffle. United States Fish and Wildlife Service, Special Scientific Report, Fisheries $n^{\circ} 405,1-11$. 